\title{
Tumor inactivation of E-cadherin: a new tool for breast cancer treatment?
}

\author{
Giovanni Corso $^{1,2}$, Bernardo Bonanni ${ }^{3}$, Paolo Veronesi ${ }^{1,2}$ \\ ${ }^{1}$ Division of Breast Surgery, European Institute of Oncology IRCCS, Milan, Italy; ${ }^{2}$ Faculty of Medicine, University of Milan, Italy; ${ }^{3}$ Division of \\ Cancer Prevention and Genetics, European Institute of Oncology IRCCS, Milan, Italy \\ Correspondence to: Giovanni Corso. Division of Breast Surgery, European Institute of Oncology, via G. Ripamonti 435, 20141 Milano, Italy. \\ Email: giovanni.corso@ieo.it. \\ Comment on: Horne HN, Oh H, Sherman ME, et al. E-cadherin breast tumor expression, risk factors and survival: Pooled analysis of 5,933 cases from \\ 12 studies in the Breast Cancer Association Consortium. Sci Rep 2018;8:6574.
}

Submitted Aug 16, 2018. Accepted for publication Aug 24, 2018.

doi: $10.21037 / \mathrm{atm} .2018 .08 .45$

View this article at: http://dx.doi.org/10.21037/atm.2018.08.45

E-cadherin protein has a critical role for establishing and maintaining polarized and differentiated epithelia through intercellular adhesion complexes.

This molecule is considered an invasion suppressor, and its deregulation is often found in advanced cases of some epithelial carcinoma (1).

Deletion or deregulation of E-cadherin is correlated with the infiltrative and metastatic ability of the tumor, believed to be because of disruption of the cadherin-catenin complex with the consequent loss of cell adhesion and concomitant increase in cell motility $(2,3)$.

In clinical practice, E-cadherin expression is evaluated to confirm lobular histopathology in breast cancer. Lobular breast tumors with low or absent E-cadherin expression are associated with high hormone receptors expression (estrogen and progesterone receptors) (4).

However, E-cadherin in not only a target to distinguish ductal carcinoma from lobular breast cancer. This obsolete concept should be abandoned.

In fact, a recent multicentric study on 5,933 female invasive breast cancers, demonstrated that E-cadherin low expression is significantly associated with poorly differentiated tumors, high tumor size volume $(>\mathrm{T} 2)$, and HER2 negative expression (5).

The association with larger and low-grade tumors is due to the aggressiveness pattern of breast tumor in absence of E-cadherin function.

Instead the complex E-cadherin /HER2 is an important emerging molecular target for breast cancer care. Other study demonstrated that in lobular breast cancer E-cadherin performs heterodimers with HER2 receptor (6). In particular, the presence of E-cadherin plus ERBB2 somatic mutations leads to a worse prognosis in lobular breast cancer (7). Instead the E-cadherin/HER2 negative association is not associated with breast cancer specific survival (5). This synergic effect of E-cadherin/ERBB2 complex is still not well clarified. It seems that inactivation of E-cadherin leads to an over-expression of HER2 with a worse impact on cancer prognosis. E-cadherin/HER2 heterodimer complex represents valid biomarkers for targeted therapies against breast cancer, never consider to date.

\section{Acknowledgements}

Funding: This manuscript was supported by the project "Understanding how CDH1 germline mutations affect hereditary lobular breast cancer", Italian Ministry of Health, grant number GR-2016-02361655.

\section{Footnote}

Conflicts of Interest: The authors have no conflicts of interest to declare.

\section{References}

1. Takeichi M. Cadherins in cancer: implications for invasion and metastasis. Curr Opin Cell Biol 1993;5:806-11.

2. Christofori G, Semb H. The role of the cell-adhesion 
molecule E-cadherin as a tumour-suppressor gene. Trends Biochem Sci 1999;24:73-6.

3. Van Aken E, De Wever O, Correia da Rocha AS, et al. Defective E-cadherin/catenin complexes in human cancer. Virchows Arch 2001;439:725-51.

4. Wasif N, Maggard MA, Ko CY, et al. Invasive lobular vs. ductal breast cancer: a stage-matched comparison of outcomes. Ann Surg Oncol 2010;17:1862-9.

5. Horne HN, Oh H, Sherman ME, et al. E-cadherin breast tumor expression, risk factors and survival: Pooled analysis

Cite this article as: Corso G, Bonanni B, Veronesi P. Tumor inactivation of E-cadherin: a new tool for breast cancer treatment? Ann Transl Med 2018;6(Suppl 1):S6. doi: 10.21037/ atm.2018.08.45 of 5,933 cases from 12 studies in the Breast Cancer Association Consortium. Sci Rep 2018;8:6574.

6. Ping Z, Siegal GP, Harada S, et al. ERBB2 mutation is associated with a worse prognosis in patients with $\mathrm{CDH} 1$ altered invasive lobular cancer of the breast. Oncotarget 2016;7:80655-63.

7. Corso G, Veronesi P, Sacchini V, et al. Prognosis and outcome in CDH1-mutant lobular breast cancer. Eur J Cancer Prev 2018;27:237-8. 